\title{
High-Throughput Single Particle Cryo-Electron Microscopy Applied to the 26S Proteasome
}

\author{
S. Nickell*, F. Beck*, E. Sakata*, A. Korinek**, W. Baumeister* and J.M. Plitzko* \\ * Max Planck Institute of Biochemistry, Dept. of Molecular Structural Biology, D-82152 \\ Martinsried, Germany \\ ** Health Sciences Centre, Dept. of Biochemistry and Biomedical Sciences, McMaster University \\ Hamilton, ON, L8N3Z5 Canada
}

The $26 \mathrm{~S}$ proteasome is a molecular machine with a key role in intracellular protein degradation in eukaryotes. The holocomplex is formed by a barrel-shaped core particle $(\mathrm{CP})$ - the $20 \mathrm{~S}$ proteasome which is capped at both ends by a regulatory particle (RP) each. While the structure of the CP has been determined already a while ago by X-ray crystallography [1], structure determination of the RP or the entire $26 \mathrm{~S}$ holocomplex poses a big challenge in structural biology. Some reasons hampering structural investigations are: (i) the sheer size of $2.5 \mathrm{MDa}$ of the holocomplex. (ii) it's fragility $-26 \mathrm{~S}$ proteasomes tend to dissociate during sample preparation and (iii) the structural variability of the intact complex. While X-ray crystallography is intrinsically depending on high levels of sample purity and homogeneity, cryo-electron microscopy of single particles can tolerate a fair degree of sample heterogeneity. This is achieved by applying smart image-classification strategies allowing a post-purification step in the computer. Prerequisite for such an in-silico sorting procedure is a large number of particle images to start with. Typically tens of thousands or even hundreds of thousands high-resolution particle images of consistent quality are required for classification and to calculate three-dimensional electron density maps.

In recent years we have gradually refined $[2,3]$ the structure of the $26 \mathrm{~S}$ proteasome to a current resolution of approximate $9 \AA$ (at 0.5 Fourier shell correlation threshold), which allowed first insights into its molecular architecture. Since sample preparations of 26S proteasomes are quite heterogeneous a high-throughput single particle data acquisition approach was pivotal to generate large data sets for an exhaustive classification [4,5].

In this communication we demonstrate the capacity of our new data acquisition pipeline applied to the $26 \mathrm{~S}$ proteasome. In conjunction with instrumentation combining the latest technological achievements in electron optics, cryogenics and robotics, namely a Titan Krios microscope (FEI Company, Eindhoven, The Netherlands) equipped with a robotic sample loading and transfer system and with a 8k (8192x8192 pixel-array) Tietz CCD camera (TVIPS GmbH, Gauting, Germany) - we were able to gather data sets of unprecedented size and quality under precisely controlled conditions. This is especially important for less well behaved samples such as the $26 \mathrm{~S}$ proteasome, where the molecules of interest are so sparse that a single frame contains only a few of them.

\section{References}

[1] M. Groll et al., Nature 1997, 386:463-471.

[2] S. Nickell et al., PNAS 2009, 106:11943-11947.

[3] S. Bohn et al., PNAS 2010, 107:20992-20997.

[4] S. Nickell et al., FEBS Letters 2007, 581:2751-2756

[5] A. Korinek et al., Microscopy and Microanalysis 2010, 16:858-859

[6] This research was supported by the European Commission in the 7th Framework Program (grant agreement HEALTH-F4-2008-201648/PROSPECTS). 